\title{
Deformation Prediction of a Deep Foundation Pit Based on the Combination Model of Wavelet Transform and Gray BP Neural Network
}

\author{
Qiang Liu $\mathbb{D}^{1},{ }^{1}$ Chun-Yan Yang, ${ }^{1}$ and Li Lin ${ }^{2}$ \\ ${ }^{1}$ College of Harbour and Coastal Engineering, Jimei University, Xiamen 361021, China \\ ${ }^{2}$ College of Civil Engineering, Fuzhou University, Fuzhou 350007, China
}

Correspondence should be addressed to Qiang Liu; liutanq007@aliyun.com

Received 17 August 2021; Revised 30 October 2021; Accepted 18 November 2021; Published 7 December 2021

Academic Editor: Chuanliang Yan

Copyright (c) 2021 Qiang Liu et al. This is an open access article distributed under the Creative Commons Attribution License, which permits unrestricted use, distribution, and reproduction in any medium, provided the original work is properly cited.

The purpose of this study was to predict the deformation of a deep foundation pit based on a combination model of wavelet transform and gray BP neural network. Using a case of a deep foundation pit, a combination model of wavelet transform and gray BP neural network was used to predict the deformation of the deep foundation pit. The results show that compared with the traditional gray BP neural network model, the relative error of the combination model of wavelet transform and gray BP neural network was reduced by $2.38 \%$. This verified that the combined model has high accuracy and reliability in the prediction of foundation pit deformation and also conforms to the actual situation of the project. The research results can provide a valuable reference for foundation pit deformation monitoring.

\section{Introduction}

At present, with the vigorous development of large-scale infrastructure in China, the excavation process of foundation pits will not only cause the deformation of foundation pits themselves, but also cause the deformation and displacement of adjacent buildings, rail transit, and underground comprehensive pipe corridors. In serious cases, it will not only cause huge economic losses, but also bring serious social consequences $[1,2]$. Therefore, how to establish a suitable deformation prediction model to monitor the excavation deformation of the foundation pit and ensure the construction safety of the excavation process plays an important role. However, because there are many influencing factors of deformation in the process of foundation pit excavation construction, and since these factors have randomness and uncertainty, it is difficult for this to be expressed accurately with a simple mathematical model [3-5]. For this reason, many experts and scholars have put forward many prediction methods for the research of foundation pit excavation deformation prediction models.
Recently, the prediction methods for foundation pit deformation mainly include artificial neural networks, gray correlation degree methods, fuzzy comprehensive evaluations, Kalman filtering models, and hybrid combination models [6-8].

However, the above methods have achieved certain results in the deformation prediction of foundation pit, but there are also shortcomings. The convergence speed of artificial neural network is relatively slow, which makes local optimization easy, making the prediction result error larger; the gray correlation degree theory can easily reduce the prediction accuracy of the model when dealing with the situation of large data fluctuation; the fuzzy comprehensive evaluation model still has great difficulty in choosing which membership function. Therefore, many scholars also try to use the hybrid combination prediction model to predict the deformation of foundation pits. Xu et al. [9] made use of the fact that the stability of the signal data decomposed by wavelet transform is better than that of the original signal and established the combination model of wavelet gray series and parallel series, respectively, to effectively predict 
the deformation of foundation pits. Liu et al. $[10,11]$ used the combination of gray model and BP neural network to predict the deformation of deep foundation pits under the condition of few samples and insufficient information, but the final fitting results were too different from the measured results. Zheng et al. [12] established the prediction model of a wavelet BP neural network. Through the comparison with the prediction results of the BP neural network model, it is concluded that the prediction results of the wavelet $\mathrm{BP}$ neural network model are more accurate than those of the BP neural network model.

The above models use the advantages of various deformation prediction models to predict the deformation of deep foundation pits using the hybrid combination model method $[13,14]$, which improves the accuracy of prediction results. In fact, foundation pit deformation is based on timeseries prediction, including the trend term and the random term [15]. Among them, the trend term reflects the main law of foundation pit deformation; it is the main basis of deformation prediction and belongs to the nonstationary series. The random term belongs to noise series and has certain stability, which is one of the main reasons affecting the accuracy of the prediction method. Therefore, these mixed combination model methods do not fully consider or distinguish the characteristics and influence of the trend term and the random term in the prediction process, and they have many shortcomings, such as being relatively simple and having their own characteristics and application occasions, so they cannot fully mine the original data information, and the prediction accuracy needs to be improved [16].

Therefore, we tried to synthesize the advantages of three kinds of deformation prediction models using a wavelet denoising to remove and impute the outliers of the original signal data to obtain a more real and reliable data signal after denoising [17-19]. Using the GM $(1,1)$ model [20], which has a better effect in processing incomplete signals and few samples, and using the BP neural network with strong computing ability and the advantage of a good error correction ability [21, 22], the prediction model of foundation pit deformation based on wavelet denoising gray $\mathrm{BP}$ neural network was established.

In this study, a new gray BP neural network method based on wavelet denoising is proposed. The prediction model method is widely used in the deformation prediction of deep foundation pit excavation. Firstly, the effective and reliable data of foundation pit deformation were obtained by wavelet denoising; secondly, the GM $(1,1)$ model was used to predict foundation pit deformation, and the predicted value was taken as the input sample value of BP neural network; and finally, the expected output prediction result of foundation pit deformation was obtained by learning and training. The contribution of this study is twofold:

(1) The innovation of this study is to propose a new gray BP neural network method based on wavelet denoising. Compared with the traditional gray BP neural network model, this method can improve the accuracy and stability of deformation prediction of deep foundation pit, more accurately predict the future deformation of deep foundation pit, and be more in line with the engineering practice.

(2) It can provide a useful reference for deformation monitoring and prediction of deep foundation pit excavation construction in the future and ensure the safety of deep foundation pit excavation construction.

The rest of this study is organized as follows. The related work is described in Section 2. The details of the proposed combined forecasting model method are shown in Section 3. The application case evaluation is presented in Section 4. The conclusion is drawn in Section 5.

\section{Related Work}

2.1. Denoising Method of Deformation Signal Based on Wavelet Transform. Foundation pit deformation monitoring data are a signal including noise, and its mathematical expression is as follows:

$$
s(x)=f(x)+e(i), \quad i=1,2, \ldots, n,
$$

where $s(x)$ represents deformation monitoring data, which include real deformation and random noise (or system error); $f(x)$ represents the real deformation, and $e(i)$ is the random noise signal.

In the analysis of deformation monitoring data, to make the monitoring data more real, it is necessary to deal with the noise. Generally speaking, the noise signal is usually a highfrequency signal, so the real signal is a stable low-frequency signal. The denoising principle of wavelet analysis is to reduce part of $e(i)$, the noise signal, which is essentially the denoising of the signal or monitoring data $s(x)$ so that the real deformation signal $f(x)$ is separated from the signal $s(x)$. In general, this method consists of three steps: wavelet decomposition, threshold quantization, and high-frequency coefficient reconstruction. In this study, the principle of wavelet multiresolution analysis was used for signal processing [23].

Suppose that space $V_{0}$ is approximated by space $L^{2}(R)$, the deformation signal $f(x)$ is regarded as belonging to the approximate space $a$, and $L^{2}(R)$ is decomposed into orthogonal components $V_{1}$ and $W_{1}$, then space $V_{1}$ is further decomposed into orthogonal components $V_{2}$ and $W_{2}$, and so on; the approximate space $V_{0}$ of space $L^{2}(R)$ is decomposed into infinite orthogonal spaces:

$$
V_{0}=V_{1} \oplus W_{1}=V_{2} \oplus W_{2} \oplus W_{1}=\cdots=V_{N} \underset{j=1}{\oplus} W_{j},
$$

where $\oplus$ is the sum of two approximate spaces of decomposition, and then, any signal $f(t)$ can be decomposed into the sum of infinite orthogonal spaces, which can be expressed as follows: 


$$
\begin{aligned}
f(t) & =f_{N}(t)+\sum_{j=1}^{N} g_{j}(t) \\
& =\sum_{k \in Z}\left\langle f, \varphi_{N, k}(t)\right\rangle \varphi_{N, k}(t)+\sum_{j=1}^{N} \sum_{k \in Z}\left\langle f, \psi_{j, k}(t)\right\rangle \psi_{j, k}(t),
\end{aligned}
$$

where scale function $\phi_{N, k}(t)=2^{-N / 2} \phi\left(2^{-N} t-k\right)$ is the standard orthogonal basis of $V_{N}$; wavelet function $\psi_{j, k}(t)=$ $2^{-j / 2} \phi\left(2^{-j} t-k\right)$ is the standard orthogonal basis of $W_{j}$ and $L^{2}(R)$.

The whole energy of all finite signal spaces $\left(L^{2}(R)=\right.$ $\left.\oplus_{j=-\infty}^{\infty} W_{j}\right)$ is covered by the closure requirements of space $W_{j}$, which is complete and satisfies the requirements of the space $V_{j} \oplus W_{j}=V_{j-1}$.

Taking the two-level decomposition as an example, the structure of the signal wavelet multiresolution analysis tree is shown in Figure 1. In Figure 1, $S$ represents the original signal, and $A_{j}$ and $D_{j}$ represent the decomposed low-frequency signal and high-frequency signal on scale $j$, respectively. Then, the low-frequency signal is further decomposed into the approximation part of low frequency and high frequency. The relationship can be expressed as follows:

$$
S=A_{j}+\sum_{m=1}^{j} D_{m} .
$$

It is worth noting that the different choices of wavelet function and threshold processing will lead to different signal denoising effects. At present, the Daubechies (dbN) wavelet and the Symlet wavelet are commonly used for signal denoising. The Symlet wavelet is based on the improvement of the $\mathrm{dbN}$ wavelet function. Its expression is as follows:

$$
m^{0}(\omega)=\frac{1}{\sqrt{2}} \sum_{k=0}^{2 N-1} h_{k} e^{-j i \omega}
$$

The Symlet wavelet and the Daubechies wavelet have symmetry, which is suitable for image processing. Both wavelet functions can reduce phase distortion in signal analysis and reconstruction.

In this study, there are four commonly used threshold selection methods.

\subsubsection{Fixed Threshold (Sqtwolog)}

$$
\lambda=\sqrt{2 \ln N},
$$

where $N$ is the sampling length of the signal.

2.1.2. Min/Max Threshold (Minimaxi). Its principle is to minimize the maximum risk of the estimate, producing an extreme value of the minimum mean square error. Its threshold algorithm is as follows:

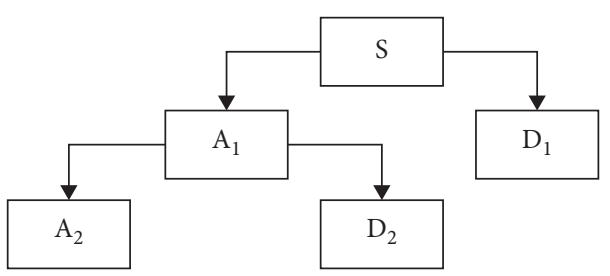

Figure 1: A tree corresponding to multiresolution analysis.

$$
\lambda= \begin{cases}0, & N \leq 32, \\ 0.3936+\frac{0.1829 \ln N}{\ln 2}, & N>32 .\end{cases}
$$

2.1.3. Unbiased Risk Estimation Threshold (Rigrsure). According to the risk curve Rish, the minimum risk point is denoted as $k_{\min }$, and then, the unbiased risk estimation threshold is defined as follows:

$$
\lambda_{k}=\sqrt{f\left(k_{\min }\right)}
$$

2.1.4. Heursure Threshold (Heursure). Heuristic threshold combines unbiased risk valuation threshold and fixed threshold, and it is defined as follows:

$$
\lambda= \begin{cases}\lambda_{\text {sqt }}, & \text { eat } \prec \text { crit }, \\ \min \left(\lambda_{\text {sqt }}, \lambda_{\text {sig }}\right), & \text { other, }\end{cases}
$$

where $\quad$ crit $=\sqrt{(1 / N)(\ln N / \ln 2)^{3}} \quad$ and $\quad$ eat $=(1 / N)$ $\left[\sum_{j=1}^{N}\left|S_{j}\right|^{2}-N\right]$.

If we can choose an appropriate threshold to denoise the signal containing noise by the threshold method, then we can achieve the purpose of denoising without losing truth.

To measure the effect of signal denoising, we adopted two quality evaluation methods. The first method is the rootmean-square error method (RMSE), which refers to the mean square error of the decomposed and reconstructed signal and the original signal. Its expression is as follows:

$$
\text { RMSE }=\sqrt{\frac{1}{N} \sum_{i=1}^{n}[f(i)-\widehat{f}(i)]^{2}},
$$

where $N$ is the signal length, $F(i)$ is the original signal, and $\widehat{f}(i)$ is the signal after wavelet denoising. RMSE represents the difference between the original signal and the denoised signal. In practical application, when the RMSE value is larger, the denoised signal has a low approximation to the original signal, and the denoising effect is poor; otherwise, the denoising effect is good.

Another method is the signal-to-noise ratio (SNR) method, which is the ratio of signal value to noise value. It is generally believed that the higher the SNR, the better the denoising effect. It is defined as follows: 


$$
\mathrm{SNR}=10 \times \lg \left(\frac{p_{s}}{p_{n}}\right),
$$

where $P_{S}$ is the power of the original signal and $P_{n}$ is noise power.

2.2. The Gray Modeling Method. The gray GM $(1,1)$ model is a common prediction model, which is composed of firstorder differential equations of first-order variables. Assume the original discrete sequence is as follows:

$$
x^{(0)}=\left\{x^{(0)}(1), x^{(0)}(2), \ldots, x^{(0)}(n)\right\} .
$$

A new sequence is generated by accumulating a sequence at a time:

$$
x^{(1)}=\left\{x^{(1)}(1), x^{(1)}(2), \ldots, x^{(1)}(n)\right\} .
$$

Further, the first-order differential equation is established for equation $(13)$, and the gray GM $(1,1)$ model is as follows:

$$
\frac{\mathrm{d} x^{(1)}}{\mathrm{d} t}+a x^{(1)}=u \text {, }
$$

where $a$ and $u$ are gray parameters, which can be solved by the least-squares method:

$$
\widehat{a}=[a, u]=\left(B^{T} B\right) B^{T} y_{n},
$$

where

$$
\begin{aligned}
& B=\left[\begin{array}{cc}
-\frac{1}{2}\left(x^{(1)}(2)+x^{(1)}(2)\right) & 1 \\
-\frac{1}{2}\left(x^{(1)}(3)+x^{(1)}(2)\right) & 1 \\
\ldots & \ldots \\
-\frac{1}{2}\left(x^{(1)}(n)+x^{(1)}(n-1)\right) & 1
\end{array}\right], \\
& y=\left[\begin{array}{c}
x^{(0)}(2) \\
x^{(0)}(3) \\
\vdots \\
x^{(0)}(n)
\end{array}\right] .
\end{aligned}
$$

After solving $\widehat{a}$, the result is substituted into equation (14) to obtain:

$$
\widehat{x^{(1)}}(k+1)=\left(x^{(0)}(1)-\frac{u}{a}\right) e^{-a k}+\frac{u}{a} .
$$

For cumulative generation, the prediction model is restored to:

$$
\widehat{x^{(0)}}(k+1)=\widehat{x^{(1)}}(k+1)-\widehat{x^{(1)}}(k)
$$

where $\hat{x}^{(0)}(2), \ldots, \hat{x}^{(0)}(n)$ is the signal data part for back testing; $\hat{x}^{(0)}(n+1), \hat{x}^{(0)}(n+2), \ldots$ is the data part of the predicted signal.

Finally, the residual and relative residual are calculated as follows:

$$
\begin{aligned}
& e\left(k_{i}\right)=x^{(0)}\left(k_{i}\right)-\hat{x}^{(0)}\left(k_{i}\right), \\
& q\left(k_{i}\right)=\frac{e\left(k_{i}\right)}{x^{(0)}\left(k_{i}\right)} .
\end{aligned}
$$

The accuracy of the gray GM $(1,1)$ model can be evaluated by the posterior variance ratio and small error probability. The posterior variance ratio and small error probability are, respectively:

$$
\begin{aligned}
& C=\frac{S_{2}}{S_{1}}, \\
& P=P\left(\left|e(k-\bar{e}) \prec 0.6745 S_{1}\right|\right),
\end{aligned}
$$

where $S_{1}$ and $S_{2}$ are the variances of model sequence $\widehat{x}^{(0)}(k)$ and residual sequence $e^{(0)}(k)$, respectively. $\bar{e}$ is the mean value of $e^{(0)}(k)$.

The smaller the $C$, the more discrete the original sequence, and the larger the variance; the smaller the residual, the higher the accuracy of the simulation model. The model accuracy level can be expressed as follows: the level of Max $\{P$, the level of $C\}$, as shown in Table 1 .

2.3. BP Neural Network Model. A BP neural network is a multilayer feedforward network with error propagation (the sum of squares of network error reaches the minimum value). A BP neural network model is shown in Figure 2.

Assume the following: input node $x_{i}$, hidden node $y_{j}$, output node $z_{k}$, network connection weight $w_{i j}$ of input node and hidden layer node, and network connection weight $v_{j k}$ of hidden layer node and output node. The expected value of the output node is $t_{k}$.

The output of hidden layer node is as follows:

$$
y_{j}=\int\left(\sum_{i} w_{i j} x_{i}-Q_{j}\right)=f\left(\text { net }_{j}\right) .
$$

The calculated output of the output node is as follows:

$$
z_{k}=\int\left(\sum_{j} v_{j k} y_{j}-Q_{k}\right)=f\left(\text { net }_{k}\right) \text {, }
$$

where net ${ }_{j}=\sum_{i} w_{i j} x_{i}-Q_{j}$ and net $_{k}=\sum_{j} v_{j k} y_{j}-Q_{k}$

The error of the output node is as follows:

$$
E=\frac{1}{2} \sum_{k}\left(t_{k}-z_{k}\right)^{2} .
$$

If $E$ meets the requirements, the sample meets the expected output; if $E$ does not meet the requirements, the network connection weight needs to be corrected. 
TABLE 1: Model accuracy grade and evaluation.

\begin{tabular}{lcc}
\hline Model accuracy & $P$ & $C$ \\
\hline Level 1 (good) & $0.95 \leq P$ & $C \leq 0.35$ \\
Level 2 (qualified) & $0.8 \leq P<0.95$ & $0.35<C \leq 0.5$ \\
Level 3 (general) & $0.7 \leq P<0.8$ & $0.5<C \leq 0.65$ \\
Level 4 (unqualified) & $P<0.7$ & $0.65<C$ \\
\hline
\end{tabular}

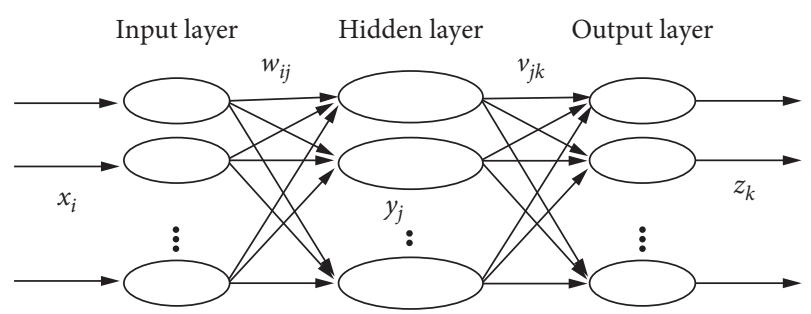

FIGURE 2: BP neural network model.

$$
\begin{array}{r}
\Delta v_{j k}=-\eta \frac{\partial E}{\partial_{v_{j k}}}=\eta \delta_{k} y_{j}, \\
v_{j k}(k+1)=v_{j k}(k)+\Delta v_{j k}, \\
\Delta w_{i j}=-\eta \frac{\partial E}{\partial_{w_{i j}}}=\eta \delta_{j} x_{i}, \\
w_{i j}(k+1)=w_{i j}(k)+\Delta w_{i j},
\end{array}
$$

where $\delta_{k}=-\left(t_{k}-z_{k}\right) f^{\prime}\left(\right.$ net $\left._{k}\right), \delta_{j}=f^{\prime}\left(\right.$ net $\left._{j}\right) \sum_{k} \delta_{k} v_{j k}, \eta$ is the training times, and $\alpha$ is the momentum factor.

To improve the generalization ability of BP network, we adopted the early termination method. At the same time, the foundation pit monitoring data were divided into three subsets: the first subset was the training sample set, which was used to calculate the gradient and correct the weight and threshold of the network. The second subset was the validation sample set, which was used to minimize process fitting and monitor the error of the validation sample set during the training process. The third subset was the test sample set, which was used to test the final solution to confirm the actual predictive ability of the network.

This study studied the use of a three-layer BP neural network (input layer, hidden layer, and output layer composition structure) for foundation pit deformation prediction. The specific selection of the parameters of the foundation pit deformation prediction model was to use the previous 12 monitoring data to construct training samples. If there were too many nodes in the input layer, the calculation cycle and workload would increase, and it would also have a certain impact on the prediction accuracy of the model. Therefore, the first $n-4$ displacement values of the original data were used as the training sequence of the BP neural network, and the last four actual measurements were used as the predicted values (verification samples). Certain monitoring data of the designated measuring point were the output, and the eight monitoring data before the measuring point were the input. The eight input data and one output data of the measuring point constituted a training sample. In this way, 11 sets of training samples could be constructed using the previous 12 monitoring data.

The BP neural network model of foundation pit deformation prediction had 8 input variables and 1 output variable, and the hidden layer had a three-layer network model with 11 nodes. On the premise that the training accuracy could be guaranteed, it was enough to ensure that the hidden layer node was larger than the input layer node; otherwise, it would affect the training speed. In the calculation of the network topology, the tansig transfer function was used to build the network, the gradient descent training was used as the training function, the training accuracy was set to 0.0001 , and the maximum number of training steps was 10 000. The training simulation calculation was performed to obtain the corresponding fitting value of the foundation pit deformation.

\section{Proposed Combination Model of Wavelet Transform and Gray BP Neural Network}

Based on wavelet analysis and gray BP neural network modeling method, we constructed a series combination model of wavelet transform and gray BP neural network. The series combination model uses the gray model to predict the data sequence after wavelet denoising and then takes the predicted value as the input sample value of BP neural network. After learning and training, the expected output prediction result is finally obtained. The specific modeling steps are as follows:

(1) After preprocessing the original data sequence, the signal data sequence after wavelet denoising is obtained.

(2) The processed signal data are judged to see whether they meet the conditions of gray modeling, and the nonconformed signal data series is transformed.

(3) The signal data after denoising are predicted by the gray GM $(1,1)$ model.

(4) According to the characteristics of the BP neural network, the prediction value of the gray GM $(1,1)$ model is taken as the input signal data sequence of the network.

(5) By learning and training BP neural network, the predicted value of the expected output is finally obtained.

In the second point of the above steps, it is worth noting that the purpose of modeling was deformation prediction. To improve the simulation accuracy of post-prediction, it was necessary to delete old information and add new information consecutively; in particular, when the quantitative change accumulated to a certain degree and qualitative change occurred, we removed the old data that could no longer reflect the current characteristics of the system. Therefore, from the perspective of prediction, the metabolic model was the most ideal prediction model.

After appropriate changes, the expression of the GM $(1,1)$ model can be written as follows: 


$$
x^{(0)}(k)=\left(\frac{1-0.5 a}{1+0.5 a}\right)^{k-2}\left(\frac{b-a x^{(0)}(1)}{1+0.5 a}\right) ; \quad k=2,3, \ldots, n .
$$

Considering the accuracy of fitting and prediction, the applicable conditions of the model are as follows [24]:

When $-a<-0.5$, the GM $(1,1)$ model is used for medium- and long-term prediction. When $0.5<-a-<0.8$, the GM $(1,1)$ model can generally be used for short-term forecasting. When $0.8<-a<-1$, the GM $(1,1)$ model should use residual correction. When $-a>1$, the GM $(1,1)$ model should not be used.

The gray modeling feasibility test was performed on the original data series, generally including grade ratio test, smoothness, and smoothness ratio test.

First, the grade ratio test is carried out for a given data sequence: $x^{(0)}=\left\{x^{(0)}(1), x^{(0)}(2), \ldots, x^{(0)}(n)\right\}$, and the grade ratio is calculated as follows: $\sigma(k)=\left(x^{(0)}(k-1) / x(k)\right)$. If $\sigma(k) \in\left(e^{2 / n}, e^{2 /(n+1)}\right)$ is true for $k=2,3, \ldots, n$, it is considered that the original data sequence $x^{(0)}=\left\{x^{(0)}(1), x^{(0)}(2), \ldots\right.$, $\left.x^{(0)}(n)\right\}$ can be used for GM $(1,1)$ modeling and gray series prediction.

Secondly, the smoothness and smoothness ratio of data series $x^{(0)}$ are tested; the condition for $x^{(0)}$ to be a smooth discrete function is as follows: $\left(x^{(0)}(k) /\right.$ $\left.\sum_{i=1}^{k-1} x^{(0)}(i)\right)=\left(x^{(0)}(k-1) / x^{(1)}(k)\right)<\varepsilon$, and $\varepsilon$ is as small as possible.

If the original data sequence $x^{(0)}$ can meet the conditions of the grade ratio test, smooth ratio, and smoothness test, it can be modeled in gray. If the above conditions are not met, the gray modeling conditions are not met, and the data need to be transformed. Then, the rank ratio of the transformed sequence of the original data sequence $x^{(0)}$ must be within the coverage range.

In this study, the horizontal displacement data of foundation pit pressure were used as the input data, and the initial value of the original data sequence was used as the initial value of the cumulative sequence. Because the gray model needed to be trained first, and several measured data needed to be reserved to verify the accuracy of the model, the first $n-4$ data of the original data were used as training samples, and the last four data were used to test the network. They were then substituted into the already built gray GM $(1,1)$ model for training.

The whole research process is summarized, and the technical route is given as follows (Figure 3).

\section{Application Studies}

In this section, a real-time dataset from the deformation measurement of a foundation pit is used to evaluate the effectiveness of the proposed method.

\subsection{General Situation of Deep Foundation Pit Engineering and} Description of Monitoring Data. The basement of the project was two floors, the excavation depth of the foundation pit was about $10 \mathrm{~m}$, and the area of the foundation pit was about $10408 \mathrm{~m}^{2}$. The foundation pit is located at main traffic arteries, and the excavation of the foundation pit has a safety impact on the surrounding buildings and roads. The site survey results of the foundation pit showed that the main soil layers within the influence range of the excavation depth of the foundation pit on the site were as follows: (1) miscellaneous fill, with a thickness of 3.50-6.10 m; (2) silt, with a thickness of 3.50-14.30 m; (3) silty soil, with a thickness of $1.70-5.30 \mathrm{~m}$; (4) silt (including mud), with a thickness of $2.90-15.10 \mathrm{~m}$; (5) silty clay, with a thickness of $1.20-3.60 \mathrm{~m}$; and (6) coarse medium sand (including mud), with a thickness of $8.00-12.30 \mathrm{~m}$.

The hydrological conditions within the influence range of the excavation depth of the foundation pit were the following: the aquifer in the site was mainly the pore confined water in layers (3) and (5). The confined water level was about $3.73 \mathrm{~m}$, and the permeability coefficient was $K_{m}=10.23 \mathrm{~m} / \mathrm{d}$. The buried depth of the stable water level of the borehole measured during the survey was about $2.50 \mathrm{~m}$.

The supporting structure of foundation pit was a single row of SMW piles with two concrete supports. The first support bottom elevation was $-3.20 \mathrm{~m}$, and the second support bottom elevation was $-7.7 \mathrm{~m}$. The protection level of foundation pit was level I. In this project, 18 horizontal displacement observation points (S1-S18) were arranged along the edge of the support structure to measure the horizontal displacement (pile top). The layout of the measurement points is shown in Figure 4. The observation time was 152 days. The whole construction process of the foundation pit excavation was divided into the following four working conditions:

(1) The first working condition: initial stress solution of undisturbed soil and initial displacement return to zero (30 days)

(2) The second working condition: construction of pile foundation enclosure, excavation of the first layer of soil (the thickness of the soil layer was $1 \mathrm{~m}$ ), construction of purlin, and the first concrete support (52 days)

(3) The third working condition: construction of the second concrete support and excavation of the second soil layer (the thickness of the soil layer was $5 \mathrm{~m})$ (40 days)

(4) Fourth working condition: excavation of the third layer of soil (the thickness of the soil layer was $4 \mathrm{~m}$ ) to the bottom of the foundation pit (30 days)

Because the space was limited, and the middle part of the long side of the foundation pit was in a weak position, its monitoring data played a control role in the deformation of the support structure. Therefore, to verify the effectiveness of the method in this study, the S18 point was selected as the research object. The original records of horizontal displacement observation of pile foundation are shown in Table 2.

It is worth noting that to ensure the reliability of the actual monitoring data of the deformation of the foundation pit, in the model prediction and processing stage, the network model adopted a multistep rolling real-time prediction 


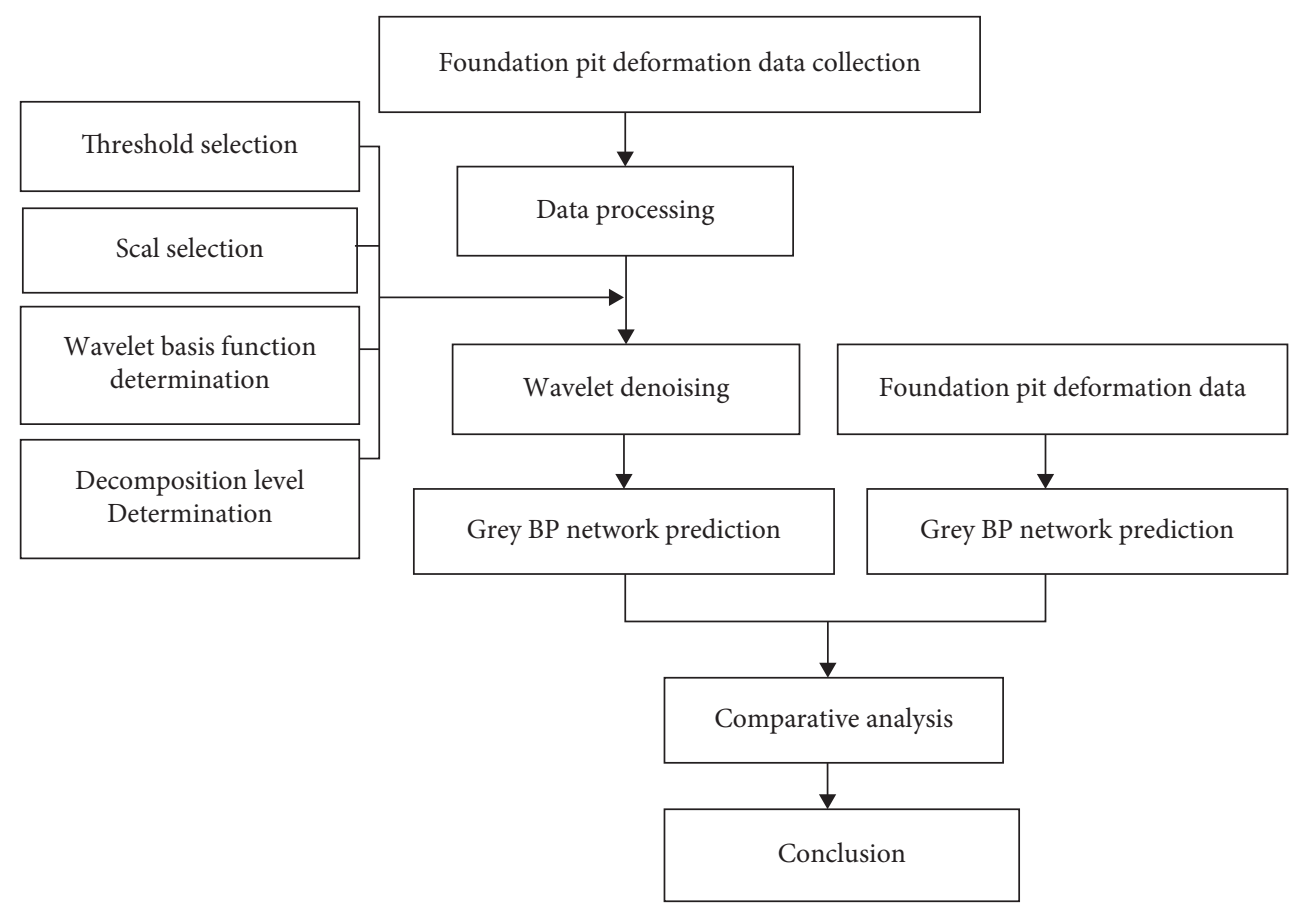

FIGURE 3: Technical route of the research process.

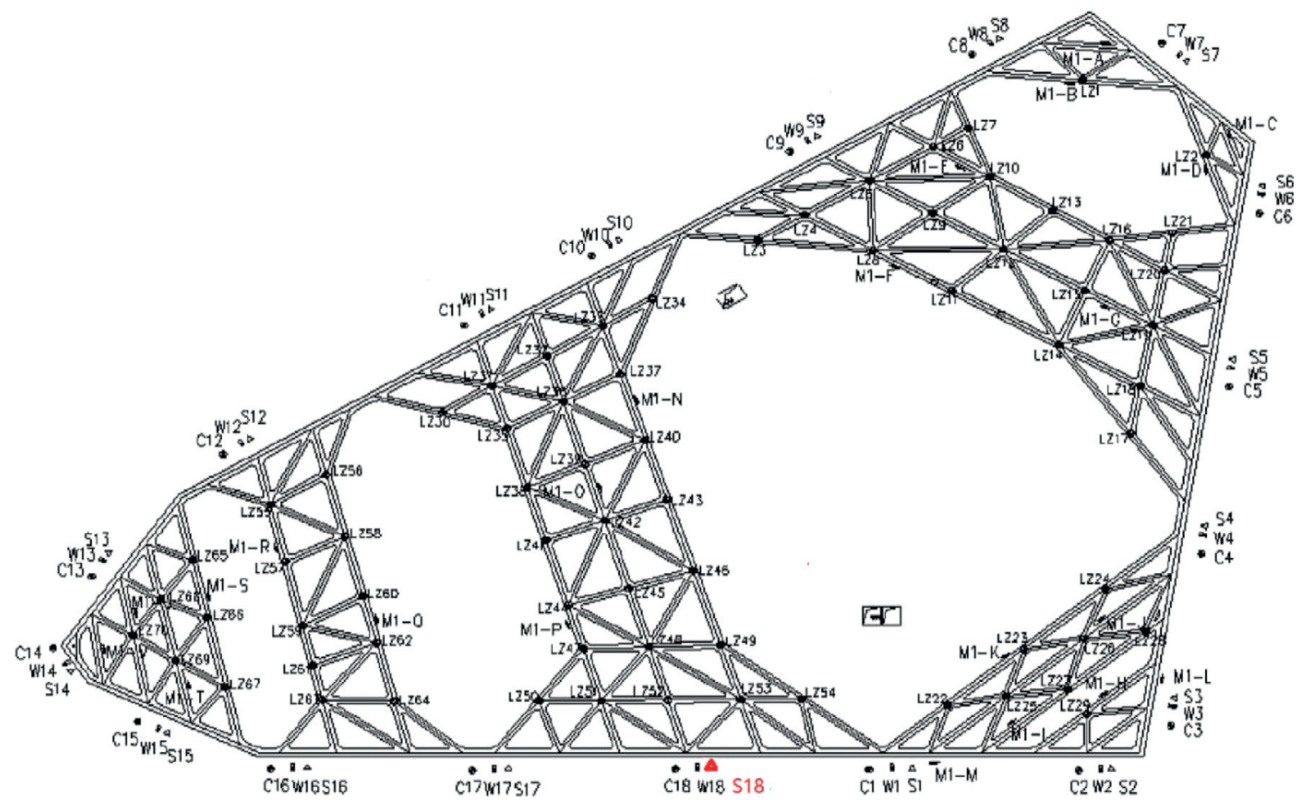

FIGURE 4: Layout of foundation pit of monitoring points.

method, taking the current horizontal displacement of the pile top as the new input and replacing the horizontal displacement of the previous period; this method was used to continuously replace the input data, and it carried out a rolling dynamic forecast for the next time. In addition, the GM $(1,1)$ model uses the original certain information to predict and analyze unknown information. Therefore, it is more sensitive to the original data. According to the new information principle, we know that the more newly acquired signals have the smallest noise error. At the same time, a single-sequence GM $(1,1)$ model can be constructed according to the time-accumulated deformation, and to meet the prediction requirements of the GM $(1,1)$ model, the data series must be positive. Therefore, this study presents another expression for the horizontal deformation data measurement, which is expressed in terms of cumulative horizontal displacement and is beneficial in reducing the error; this is suitable for the original data sequence with a small number of calculations and a small sample, and the calculation accuracy can also meet the requirements. 
TABle 2: Data of measured horizontal displacement of pile foundation and wavelet denoising ( $\mathrm{mm}$ ).

\begin{tabular}{lccc}
\hline Measured period & Time interval & Measured displacement & Displacement (wavelet denoising) \\
\hline 1 & 30 & 0.64 & 0.62 \\
2 & 13 & 1.25 & 1.21 \\
3 & 13 & 1.84 & 1.89 \\
4 & 13 & 2.36 & 2.32 \\
5 & 13 & 2.65 & 2.59 \\
6 & 10 & 3.83 & 2.93 \\
7 & 10 & 4.67 & 4.56 \\
8 & 10 & 5.38 & 5.31 \\
9 & 10 & 6.45 & 6.51 \\
10 & 10 & 6.67 & 6.58 \\
11 & 10 & 6.78 & 6.81 \\
12 & 10 & 6.87 & 6.91 \\
\hline
\end{tabular}

\subsection{Results and Discussion}

4.2.1. Horizontal Displacement Data (Pile Top) Based on Wavelet Denoising. A different selection of wavelet function and threshold processing will lead to different denoising effects. In this study, the threshold value and the different parameters of wavelet basis function were selected to make a comparative study of the data effect after denoising, and the best model of wavelet denoising for the horizontal displacement of S18 point pile top was determined, and then, the next stage of analysis and prediction was carried out.

For the evaluation of a different threshold selection, unified scale $=$ SLN, soft threshold, Daubechies' 3 wavelets, and one-level decomposition were used. A fixed threshold (sqtwolog), unbiased risk estimation threshold (rigrsure), and heuristic threshold (heursure) were used to denoise the original data, and different filtering effects were obtained. The evaluation results of three different threshold selections are shown in Table 3.

According to the comparison of Table 3, in relation to the definition of root-mean-square error (RMSE) and the signalto-noise ratio (SNR), the smaller the RMSE value, the higher the approximation between the denoised signal and the original signal, and the better the denoising effect; the larger the signal-to-noise ratio, the better the denoising effect. From these two evaluation indexes, the denoising effect based on the unbiased risk estimation threshold (rigrsure) method is relatively better. Therefore, to predict more accurately, we chose the principle of rigrsure for wavelet denoising.

We performed a comparison and evaluation of different wavelet basis functions. There are many choices of wavelet basis functions, and the denoising effect of different wavelet basis functions is different. In this study, only Db6 and Sym6 wavelets with orthogonality and compactness were selected. We used the principle of the rigrsure threshold, scale $=$ SLN, and one-level decomposition to denoise the original data; the evaluation results are shown in Table 4.

It can be seen from Table 4 that the root-mean-square error of the Db6 wavelet function was relatively smaller, and the signal-to-noise ratio was larger. Therefore, Db6 was chosen as the wavelet basis function of wavelet denoising. Finally, we decided to use the Db6 wavelet function, one-
TABle 3: Denoising evaluation of three thresholds.

\begin{tabular}{lcc}
\hline Threshold principle & RMSE & SNR \\
\hline Sqtwolog & 0.786 & 27.563 \\
Rigrsure & 0.243 & 39.623 \\
Heursure & 0.412 & 35.426 \\
\hline
\end{tabular}

TABLE 4: Denoising evaluation of two wavelet functions.

\begin{tabular}{lcc}
\hline Wavelet functions & RMSE & SNR \\
\hline Db6 & 0.316 & 38.156 \\
Sym6 & 0.493 & 33.156 \\
\hline
\end{tabular}

level decomposition, rigrsure function, and scale $=$ SLN to denoise the original observation data and obtained the denoised data as shown in Table 2.

4.2.2. Predicting the Deformation Based on the Gray BP Network Model Based on the Measured Data. According to the method introduced in the second part, the 8-step gray GM $(1,1)$ model was selected to predict the 9-12 horizontal displacement data using the measured horizontal displacement data of the pile top in the period of $1-8$. The fitting value test and prediction value test of the 8 -step gray GM $(1,1)$ model are shown in Table 5.

It can be seen from Table 5 that the posteriori difference ratio of the fitting value of the $\mathrm{GM}$ gray $(1,1)$ model was 0.31 , less than or equal to 0.35 , and its model accuracy grade was grade I; its prediction result was very good, and its reliability was high. In addition, it can be seen from the test results that, with the increase in observation periods, the relative error was also gradually increasing, mainly because the data change trend in this period suddenly slowed down, and the prediction error of the gray model itself also gradually increased with the increase in observation periods, which makes the prediction accuracy not high.

Therefore, the BP neural network prediction was carried out according to the GM $(1,1)$ fitting results. Considering the small amount of data, we took the $\operatorname{GM}(1,1)$ fitting value of the pile top horizontal displacement data from the first period to the eighth period as the input sample, took the 
TABLE 5: Test for fitting value and predicted value of horizontal displacement of pile top (mm).

\begin{tabular}{|c|c|c|c|c|c|}
\hline Measured period & Measured displacement & Fitting value & Residual & Relative error & Average error \\
\hline 1 & 0.64 & 0.64 & 0 & 0 & \multirow{8}{*}{$C=\left(S_{2} / S_{1}\right)=0.31$} \\
\hline 2 & 1.25 & 1.26 & -0.01 & $0.87 \%$ & \\
\hline 3 & 1.84 & 1.90 & -0.06 & $3.25 \%$ & \\
\hline 4 & 2.36 & 2.42 & -0.57 & $2.43 \%$ & \\
\hline 5 & 2.65 & 2.45 & 0.2 & $7.68 \%$ & \\
\hline 6 & 3.83 & 3.92 & -0.09 & $2.38 \%$ & \\
\hline 7 & 4.67 & 4.47 & 0.02 & $0.45 \%$ & \\
\hline 8 & 5.38 & 5.58 & -0.02 & $3.64 \%$ & \\
\hline \multicolumn{6}{|c|}{ Forecast value inspection form } \\
\hline Measured period & Measured displacement & Fitting value & Residual & Relative error & Average error (\%) \\
\hline 9 & 6.45 & 6.79 & -0.34 & $5.27 \%$ & \multirow{4}{*}{8.17} \\
\hline 10 & 6.67 & 7.02 & -0.35 & $5.24 \%$ & \\
\hline 11 & 6.78 & 7.39 & -0.58 & $8.53 \%$ & \\
\hline 12 & 6.87 & 7.81 & -0.94 & $13.62 \%$ & \\
\hline
\end{tabular}

TABLE 6: Prediction of gray BP network model.

\begin{tabular}{|c|c|c|c|c|c|}
\hline Measured period & Measured displacement & Fitting value & Residual & Relative error (\%) & Average error (\%) \\
\hline 9 & 6.45 & 6.78 & -0.33 & 5.12 & \multirow{4}{*}{6.70} \\
\hline 10 & 6.67 & 7.01 & -0.34 & 5.23 & \\
\hline 11 & 6.78 & 7.24 & -0.46 & 6.73 & \\
\hline 12 & 6.87 & 7.54 & -0.67 & 9.7 & \\
\hline
\end{tabular}

TABLe 7: Prediction of horizontal displacement based on gray BP network model after wavelet denoising.

\begin{tabular}{lccccc}
\hline Measured period & Measured displacement & Fitting value & Residual & Relative error (\%) & Average error (\%) \\
\hline 9 & 6.45 & 6.76 & -0.31 & 4.81 & 3.12 \\
10 & 6.67 & 6.88 & -0.19 & 3.65 & 4.32 \\
11 & 6.78 & 7.03 & -0.25 & 5.68 & \\
12 & 6.87 & 7.26 & -0.39 & & \\
\hline
\end{tabular}

ninth period to the twelfth period as the expected prediction output, and obtained the prediction results of the gray BP network model, as shown in Table 6.

It can be seen from Table 6 that the accuracy of the gray BP combined model was improved to a certain extent compared with the single gray GM $(1,1)$, and the average relative error of the final model prediction was reduced by $1.47 \%$, indicating that the prediction of the gray BP model is relatively reliable and effective.

\subsubsection{Deformation Prediction of Gray BP Neural Network} Based on Wavelet Denoising. In the same way, according to the method in the previous section, considering the small amount of data, we used the $\operatorname{GM}(1,1)$ fitting prediction result of the horizontal displacement of the pile top (wavelet denoising data) from the first period to the eighth period as the output value is used to predict the horizontal displacement of the BP neural network in periods 9-12, as shown in Table 7.

As can be seen from Table 7, with the increase in observation periods, the relative error had not simply increased. The relative error in the 10 th and 11 th periods was smaller and better, while the relative error in the 9th and 12 th periods was larger. This shows that the deformation data after wavelet denoising can better reflect the real horizontal displacement of the pile top.

4.2.4. Comparison and Analysis of Gray BP Network Prediction Model before and after Wavelet Denoising. Based on the above analysis results, the comparison between the measured data and the gray BP prediction data before and after wavelet denoising is shown in Table 8.

It can be seen from Table 8 that the prediction results of the gray BP model before and after wavelet denoising were larger than the actual observation data, which was due to a sudden change in the original observation data. From the comparison of the two groups of data, the average relative error of the prediction results of the gray BP model based on wavelet denoising was $4.32 \%$, while the average relative error of the prediction results of the gray BP neural network model based on the original monitoring data was $6.70 \%$. Therefore, for the prediction data of the S18 pile top horizontal displacement, the gray BP model based on wavelet denoising was more accurate and reliable than the data without denoising.

In addition, it can be seen from Table 8 that with the increase in excavation depth of the foundation pit, the change in horizontal displacement increased. However, 
TABLE 8: Comparison of gray BP prediction data before and after wavelet denoising.

\begin{tabular}{lccccc}
\hline Measured period & 9 & 10 & 11 & 12 & Average error \\
\hline Measured displacement & 6.45 & 6.67 & 6.78 & 6.87 & 7.57 \\
Gray BP prediction & 6.78 & 7.01 & 7.24 & $6.70 \%$ \\
Gray BP prediction of wavelet denoising & 6.76 & 6.88 & 7.03 & 7.26 & $4.32 \%$ \\
\hline
\end{tabular}

during the predicted observation period from the 9th to the 12th (during the fourth stage of construction), although the horizontal displacement value of the pile top increased gradually, the increase was relatively gentle. This is because the horizontal displacement of the pile top tended to be stable with the completion of the excavation Earth pressure release and the formation of the second support. The supporting structure system formed by retaining the pile, crown beam, and support played a good role in controlling the horizontal deformation of the pile top. All the predicted results are also in line with engineering practice.

\section{Conclusion}

This research combines the advantages of wavelet transform, a gray model, and a BP neural network prediction model. We established a gray BP neural network prediction model based on wavelet transform and conducted deformation prediction research on deep foundation pit engineering. Studies have shown that the gray BP neural network model based on wavelet transform has an average relative error of $4.32 \%$ for the prediction results of deep foundation pit deformation, which is $2.38 \%$ less than the traditional gray BP neural network model. The prediction accuracy was improved, and the reliability of the method was verified. This method can provide a useful reference for deformation monitoring and the prediction of the deep foundation pit excavation construction in the future and ensure the safety of the deep foundation pit excavation construction process.

Due to the modeling granularity, our modeling only considered some key information, but some details were not considered, such as the influence of the settlement of the foundation around the foundation pit, the change in groundwater, and the change in the internal force of the concrete internal support. However, in fact, if these random factors are considered, the relative error of deformation prediction of foundation pits will be caused. Therefore, in future work, we will study how to use other real-time monitoring data to predict the deformation of deep foundation pits. We will also apply other algorithms, including advanced learning algorithms, to search for the optimal solution more quickly. In addition, how to provide a clearer visual expression model structure to guide the safety of foundation pit excavation and construction will be another direction of future foundation pit deformation prediction research work.

\section{Data Availability}

The simulation data used to support the findings of this study are included within the article.

\section{Conflicts of Interest}

The author(s) declare(s) that there are no conflicts of interest regarding the publication of this study.

\section{Acknowledgments}

This work was supported by the Natural Scientific Research Foundation of Fujian, China (Grant No. 2021J01133206).

\section{References}

[1] W. L. Yang, Y. Hu, C. Hu, and M. Yang, "An agent-based simulation of deep foundation pit emergency evacuation modeling in the presence of collapse disaster," Symmetry, vol. 581, no. 10, pp. 691-707, 2018.

[2] A. D. Ijaz, M. Ayyab, M. Zaman, M. N. Anjum, and X. H. Dong, "Finite-difference numerical simulation of dewatering system in a large deep foundation pit at Taunsa Barrage, Pakistan," Sustainability, vol. 694, no. 11, pp. 341-357, 2019.

[3] H. Liu, H. Q. Zhang, and B. Liu, "A prediction method for the deformation of deep foundation pit based on the particle swarm optimization neural network," Journal of Jilin University, Earth Science Edition, vol. 44, no. 6, pp. 1609-1614, 2014.

[4] W. X. Wang, X. Gao, and J. H. Ding, "Deep foundation pit deformation prediction in complicated geological conditions," International Journal of Engineering and Technical Research, vol. 67, no. 8, pp. 5-12, 2018.

[5] Z. Ding, D. Wang, J. Y. Wang, and X. J. Wei, "Deformation characteristics of Zhejiang soft soil deep foundation pits and their predictive analysis," Yantu Lixue/Rock and Soil Mechanics, vol. 36, no. 8, pp. 506-512, 2015.

[6] Y. Zhao, D. Zhou, and H. Yan, "An improved retrieval method of atmospheric parameter profiles based on the BP neural network," Atmospheric Research, vol. 213, no. 6, pp. 389-397, 2018.

[7] J. Guo, X. Xiao, J. Liu, and S. Mao, "Stability of GM $(1,1)$ power model on vector transformation," Journal of Systems Engineering and Electronics, vol. 26, no. 1, pp. 103-109, 2015.

[8] L. Wang, Y. Qiang, S. H. Li, and Z. C. Yang, "Research on slope deformation prediction based on fractional-order calculus gray model," Advances in Civil Engineering, vol. 211, no. 11, pp. 1687-8086, 2018.

[9] X. J. Xu, Z. Y. Huang, C. Y. Ling, and H. Zhang, “Application of grey theory and wavelet in deformation monitoring data processing," Geotechnical Investigation \& Surveying, vol. 211, no. 4, pp. 80-83, 2014.

[10] J. Liu, M. Wu, and J. X. Yuan, "Research of deep foundation pits prediction based on combining Markov chain-grey and BP neural network model," Journal of Shantou University (Natural Science), vol. 32, no. 4, pp. 53-60, 2017.

[11] C. S. Huang and J. S. Zhang, "Forecasting model of deep foundation pits deformation based on grey Markov chain 
theory," Joural of railway science and engieerng, vol. 8, no. 1, pp. 71-75, 2011.

[12] H. Zheng, T. Huang, and Y. Liu, "Application of wavelet-BP neural network model in deformation prediction of metro," Site Investigation Science and Technology, vol. 4, no. 2, pp. 50-54, 2015.

[13] Q. Guo, G. Q. Li, C. Deng, J. Wu, Z. Y. Chen, and X. B. Xu, "Rolling bearing fault diagnosis based on wavelet packet transform and convolutional neural network," Applied Sciences, vol. 10, no. 3, pp. 70-77, 2020.

[14] C. M. Vong and P. K. Wong, "Engine ignition signal diagnosis with wavelet packet transform and multi-class least squares support vector machines," Expert Systems with Applications, vol. 38, no. 2, pp. 8563-8570, 2011.

[15] F. Xiong, Q. Jiang, and C. Xu, "Fast equivalent micro-scale pipe network representation of rock fractures obtained by computed tomography for fluid flow simulations," Rock Mechanics and Rock Engineering, vol. 54, no. 2, pp. 937-953, 2021.

[16] F. Xiong, W. Wei, C. S. Xu, and Q. H. Jiang, "Experimental and numerical investigation on nonlinear flow behaviour through three dimensional fracture intersections and fracture networks," Computers and Geotechnics, vol. 121, pp. 1-9, 2020.

[17] G.-d. Yue, X.-s. Cui, Y.-y. Zou, X.-t. Bai, Y.-H. Wu, and H.-T. Shi, "A bayesian wavelet packet denoising criterion for mechanical signal with non-Gaussian characteristic," Measurement, vol. 138, no. 6, pp. 702-712, 2019.

[18] W. L. Zeng, X. P. Fu, C. H. Hu, and Y. J. Du, "Wavelet denoising with generalized bivariate prior model," Multimedia Tools \& Applications, vol. 77, no. 3, pp. 20863-20887, 2018.

[19] T. Turker, D. Sengul, A. Moloud, E. B. Mohammad, and P. Paweł, "Face recognition with triangular fuzzy set-based local cross patterns in wavelet domain," Symmetry, vol. 787, no. 11, pp. 1-13, 2019.

[20] C. D. Ding, Z. G. Fang, C. Q. Yuan, and S. F. Liu, "On the effectiveness of GM $(1,1)$ model with different sequence sizes: an evidence from random simulations," Journal of Grey System, vol. 30, no. 3, pp. 97-115, 2018.

[21] Y. T. Liu, "Incomplete big data imputation mining algorithm based on BP neural network," Journal of Intelligent \& Fuzzy Systems, vol. 11, no. 2, pp. 1-10, 2019.

[22] J. G. Zhou, B. L. Jin, S. J. Du, and P. Zhang, "Scenario analysis of carbon emissions of Beijing-Tianjin-Hebei," Energies, vol. 11, no. 2, pp. 1-11, 2018.

[23] S. A. Chouakri, F. Bereksi-Reguig, and A. T. Ahmed, "QRS complex detection based on multi wavelet packet decomposition," Applied Mathematics and Computation, vol. 217, no. 7, pp. 9508-9525, 2011.

[24] Z.-J. Zhou and C.-H. Hu, "An effective hybrid approach based on grey and ARMA for forecasting gyro drift," Chaos, Solitons \& Fractals, vol. 35, no. 3, pp. 525-529, 2008. 